\title{
The upgrade project of the T2K near detector
}

\author{
Davide Sgalaberna* on behalf of the T2K collaboration \\ University of Geneva \\ E-mail: davide.sgalaberna@cern.ch
}

The T2K neutrino oscillation experiment established the $v_{\mu} \rightarrow v_{e}$ appearance with only $10 \%$ of the original beam request of $7.8 \times 10^{21} 30 \mathrm{GeV}$ protons on target (p.o.t.). In view of the J-PARC program of upgrades of the beam intensity, the T2K-II proposal requires to run up to $20 \times 10^{21}$ p.o.t., i.e. an increase of the exposure by more than a factor 10, aimed at establishing $\mathrm{CP}$ violation at $3 \sigma$ level if the phase $\delta_{c p}$ is near to $-\pi / 2$ and the neutrino mass ordering is normal.

The Hyper-K proposal consists of a further increase of the far detector mass by a factor 10 . Facing the potential increase of statistics by two orders of magnitude, it is of great importance to undertake a vigorous program of near detector upgrades, with the aim of reducing the overall statistical and systematic uncertainties at the appropriate level of better than $4 \%$.

Time Projection Chambers equipped with Micro-Pattern-Gas detectors (MPGD) have been used with success for the T2K ND280 near detector and are now proposed for its upgrade, together with a novel fine-grained scintillator-based target and Time-of-Flight (ToF) detectors.

A project (CERN-SPSC-2017-002 and SPSC-EOI-015) combining the upgrade of the T2K near detector and the R\&D for a High Pressure TPC has been recently launched. A High Pressure TPC would be a very sensitive detector for the detailed study of neutrino-nucleus interactions, a limiting factor for extracting the ultimate precision in long baseline experiments.

The European Physical Society Conference on High Energy Physics

5-12 July, 2017

Venice

* Speaker. 


\section{Introduction}

T2K is a neutrino long-baseline experiment that precisely measures the neutrino oscillations [1]. It is a complex 2-detector experiment performed over a baseline of several kilometres. An intense (anti)neutrino beam peaked at $600 \mathrm{MeV}$ is produced at the J-PARC proton accelerator centre in Japan and is measured in two location: a near detector (ND280), placed $280 \mathrm{~m}$ away from the neutrino source, that measures the (anti)neutrino rate and spectrum unaffected by oscillations and a far detector (Super-Kamiokande), $295 \mathrm{~km}$ away, where the oscillation probability is near maximal. The measurement of $v_{\mu} \rightarrow v_{e}$ appearance with a non-zero $\theta_{13}$ [2] has opened the possibility to look for $\mathrm{CP}$ violation in neutrino oscillations: recently $\mathrm{T} 2 \mathrm{~K}$ has observed a first hint of maximal $\mathrm{CP}$ violation $[3,4,5]$. The $\mathrm{T} 2 \mathrm{~K}$ collaboration is planning to extend the data taking program in order to collect $20 \times 10^{21}$ p.o.t., about three times with respect to the original beam request, by 2025 with the goal of searching for the first evidence of CP violation [6]. As shown in fig. 1 the phase II of the T2K experiment will be accompanied by a gradual increase of the neutrino beam intensity, up to about 1.3 MW at the end of the experiment. However in order to improve the sensitivity to search for $\mathrm{CP}$ violation, also a reduction of the systematic uncertainties is required. For this reason the $\mathrm{T} 2 \mathrm{~K}$ collaboration has planned an upgrade of the near detector.
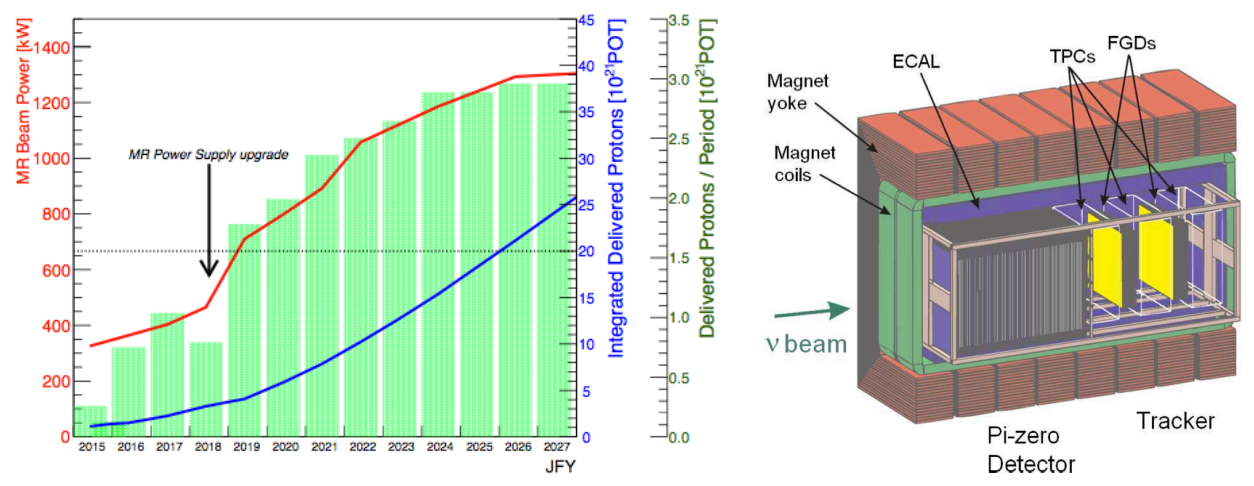

Figure 1: Left: expected beam power and p.o.t. accumulation as a function of the Japanese Fiscal Year (JFY). Right: picture of the current ND280 configuration.

The ND280 detector, shown in fig. 1, is a magnetized detector composed by a $\pi^{0}$ detector (P0D) and two fine-grained detectors (FGDs), acting as neutrino target, sandwiched between three Time Projection Chambers (TPCs). All the detectors are embedded inside an electromagnetic calorimeter (ECal). ND280 is optimized to detect particles propagating in the forward direction but not backward or perpendicular to the neutrino beam. For this reason the T2K collaboration is planning to upgrade ND280 in order to improve its acceptance. The reference design consists of a new tracker, made of a horizontal 2 tons plastic scintillator target, $(1.8$ (width $) \times 0.6$ (height $) \times$ 2(length) $\mathrm{m}^{3}$ dimension) sandwiched between two horizontal TPCs above and below it, as shown in fig. 2. The new detectors will be surrounded by a Time-of-Flight (ToF) detector, to precisely measure the sense of the tracks, reject the Out-Of-Fiducial-Volume (OOFV) background and improve the Particle IDentification (PID). The new detectors would be installed in front of the already 
existing FGDs and TPCs and would increase the total neutrino target mass up to about 4.3 tons with an almost $4 \pi$ acceptance.

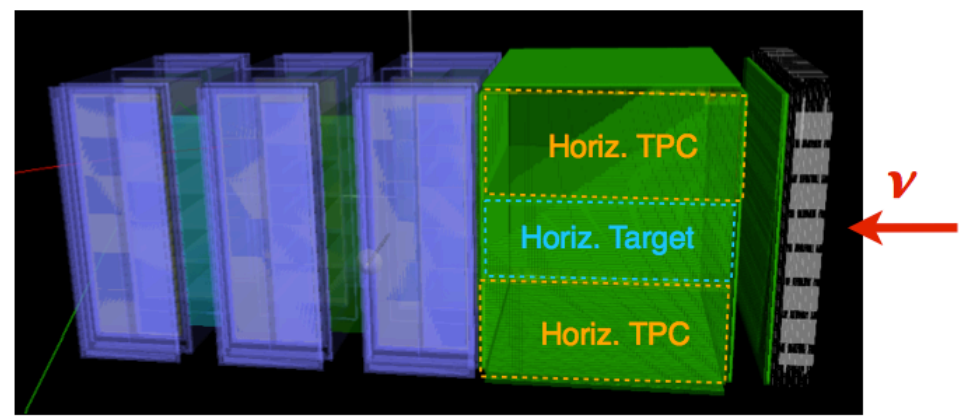

Figure 2: Picture of the upgraded ND280 configuration.

\section{The Time Projection Chambers}

The TPCs, currently installed inside ND280, use an Argon-based gas (Ar:CF4:iC4H10 95:3:2) and MicroMegas detectors [7] to read out the charge. Thanks to their excellent performances, they are key detectors for the precise measurement of the particle momentum (9\% resolution at $\sim 1 \mathrm{GeV}$ ), identification by energy loss (7.8\% resolution for minimum ionization particles) and tracking $(0.7 \mathrm{~mm}$ space resolution). We plan to build the new TPCs with similar performances. The electric field will be along the transversal direction, parallel to the ND280 magnetic field, with the cathode in the center and the anodes on the left and right planes. We plan to use resistive MicroMegas, to protect from intrinsic spark and provide a better spatial resolution [8]. Each TPC will have a drift length of $\sim 90 \mathrm{~cm}$ and about 32,000 readout channels. In order to maximize the acceptance we will build a thin field cage (about $2 \mathrm{~cm}$ ) and only a few $\%$ of a radiation length, following the scheme developed for the ILC experiment [9].

\section{The neutrino active target}

The new neutrino target will be a plastic scintillator detector, similar to the current ones. It will add about 2.3 tons to the total target mass and will provide both the neutrino vertex position and the particle tracking. The goal of the new target is to detect particles produced at any angle, not just forward as for the FGDs (plastic scintillator bars along X-width and Y-height). A few alternative solutions are considered.

The first option is a novel plastic scintillator detector [10], called Super-FGD. As shown in fig. 3, it consists of several $1 \times 1 \times 1 \mathrm{~cm}^{3}$ cubes made of extruded plastic scintillator, with three wavelength shifting (WLS) fibers along the three orthogonal directions that read out the scintillation light and provide three views (XY, XZ and YZ). This configuration would allow to detect particles produced at any angle and at lower momenta. For instance we expect to reduce the proton momentum threshold from $\sim 450 \mathrm{MeV} / \mathrm{c}$ (FGD) to about $\sim 300 \mathrm{MeV} / \mathrm{c}$ (Super-FGD). We also 
expect to reject a large fraction of $\gamma \rightarrow e^{+} e^{-}$conversion-in-target events, the largest background to the measurement of $v_{e}\left(\bar{v}_{e}\right)$ interactions. The collaboration has started an extensive R\&D program to develop this novel technology.

A second option would consist to install another type of plastic scintillator detector, used in the WAGASCI experiment [11]. Thanks to its geometry, made of plastic scintillator bars aligned in a 3D grid (see fig. 3), it provides a $4 \pi$ acceptance. The spaces between scintillators are filled with water, the same target material of the $\mathrm{T} 2 \mathrm{~K}$ far detector.
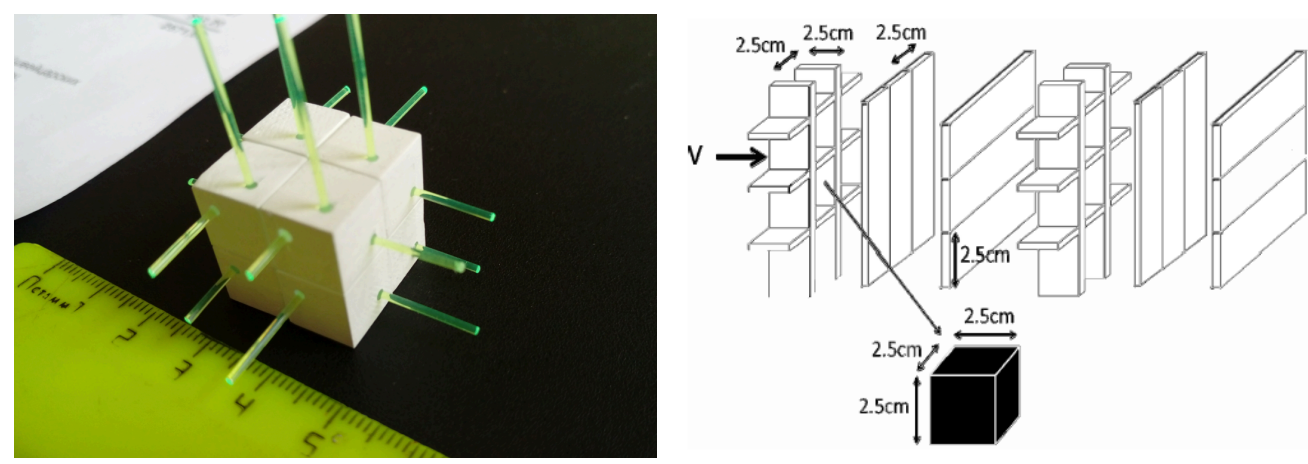

Figure 3: Left: picture of a small prototype of the Super-FGD. Right: schematic view of the 3D grid-like structure of the WAGASCI plastic scintillator bars. The spaces between scintillators are filled water.

\section{The Time-of-Flight detector}

One of the main issues in the track reconstruction is given by the determination of the sense of those tracks whose timing is not well measured. In order to provide a precise timing, a ToF detector, made of plastic scintillator bars, will embed the new detectors. Though its primary goal is to measure the sense of the tracks, it could also be used to improve the PID when the $\mathrm{dE} / \mathrm{dx}$ measurement with the TPCs cannot provide enough separation. This is the case of positrons, whose $\mathrm{dE} / \mathrm{dx}$ is similar to the one of protons at $\sim 1 \mathrm{GeV} / \mathrm{c}$, or muons with a momentum of $\sim 0.2 \mathrm{GeV} / \mathrm{c}$. In particular the first case largely contribute to the background contamination in the measurement of $\bar{v}_{e}$ interactions. The T2K collaboration is considering two different technologies.

The first option consists of a sandwich of two 20 (width) $\times 0.7$ (thickness) $\times 270$ (length) $\mathrm{cm}^{3}$ extruded plastic scintillator bars. In each bar the scintillation light would be read out at both ends by four $1 \mathrm{~mm}$ Kuraray WLS fibers, each one connected to a $3 \times 3 \mathrm{~mm}^{3}$ Multi-Pixel Photon Counter (MPPC). The measured time resolution is $\sim 630 \mathrm{ps}$, enough to precisely measure the track sense.

The second option consists of 6 (width) $\times 1$ (thickness) $\times 150$ (length) $\mathrm{cm}^{3}$ EJ-200 cast plastic scintillator bars, with an attenuation length of about $4 \mathrm{~m}[12,13]$. The scintillation light propagates through the plastic, without using any WLS fiber, and is read out at both ends with an array of eight $6 \times 6 \mathrm{~mm}^{2} \mathrm{SiPM}$. The measured time resolution is better than $100 \mathrm{ps}$. Studies are ongoing to understand the impact of both technologies on the physics measurements. 


\section{Expected performances of the upgraded ND280}

The expected performances of the upgraded detector are studied with GEANT4 [14] simulations. The expected total number of selected CC- $v_{\mu}$ events will be doubled thanks to the increased neutrino target mass. As shown in fig. 4, thanks to the upgraded configuration of the ND280 detector, the charged-current (CC) $v_{\mu}$ selection efficiency would be dramatically improved when muons are emitted at high angles or with backward direction. Analogous results were obtained for CC- $\bar{v}_{\mu}$ interaction. Thanks to the improved performance we expect to reduce the systematic uncertainties by $20-40 \%$ with respect to the current ND280 configuration.

This study takes into account only the muons detected in a TPC. However work is ongoing to study the event sample of muons stopping and detected in the neutrino target detector, with the goal to add more statistics (up to 20-30\%). In case of the Super-FGD technology the acceptance will be higher than $90 \%$ at $4 \pi$.

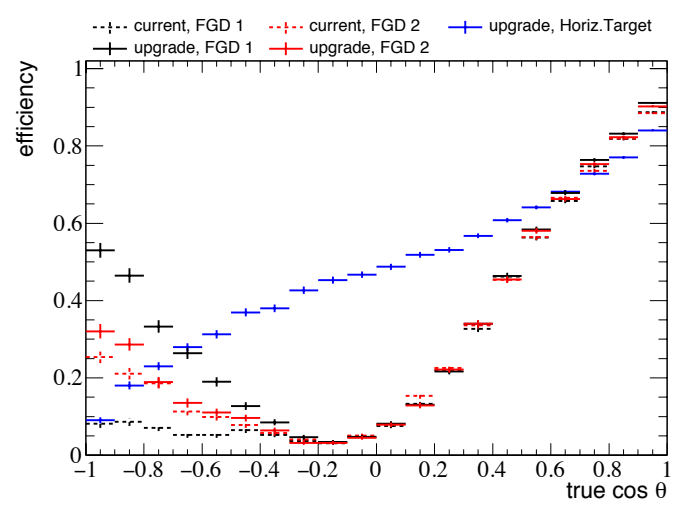

Figure 4: Selection efficiency as a function of the muon angle with respect to the longitudinal axis of CC- $v_{\mu}$ events, where the muon is detected in a TPC. The solid lines correspond to ND280 upgrade-like configuration while the dashed line correspond to ND280 current-like. The efficiency is shown for events selected in each different target detector.

\section{Conclusions}

In view of the T2K phase-II, the collaboration is working on the design of the upgraded near detector with the goal of increasing the total neutrino target mass and improving the angular acceptance, to reduce the systematic uncertainties in the measurement of neutrino oscillations. The reference design consists of a new plastic scintillator detector, acting as neutrino active target, and two horizontal TPCs. An Expression Of Interest was submitted in January 2017 to the CERN SPSC (CERN-SPSC-2017-002 and SPSC-EOI-015), signed by more than 190 physicists. The first detector prototypes will be ready in fall 2017 and 2018, when test-beam data will be collected at CERN. The T2K collaboration plans to complete the installation of the new detectors in 2021 and start the data taking in the beginning of the T2K phase-II. In parallel to the upgrade of ND280, the T2K collaboration is also working on the R\&D of a High-Pressure TPC, as a detector to explore the details of neutrino interactions. For instance the TPC gas will be the neutrino target. This technol- 
ogy will allow to precisely measure almost all the particles produced by the neutrino interactions, helping to further infer the neutrino cross-section models.

\section{References}

[1] K. Abe et. al (T2K Collaboration), Nuclear Instruments and Methods in Physics Research A 659, 106-135 (2011), 10.1016/j.nima.2011.06.067

[2] K. Abe et al. (T2K Collaboration), Phys. Rev. Lett. 107, 041801 (2011), 10.1103/PhysRevLett.107.041801

[3] K. Abe et al. (T2K Collaboration), Phys. Rev. Lett 118, 151801 (2017), 10.1103/PhysRevLett.118.151801

[4] K. Abe et al. (T2K Collaboration), arXiv:1707.01048

[5] http://www.icrr.u-tokyo.ac.jp/2017/08/04110001.html

[6] K. Abe et al. (T2K Collaboration), arXiv:1609.04111

[7] I. Giomataris, et al., Nucl. Instr. and Meth. A 560 (2006) 405, 10.1016/j.nima.2005.12.222

[8] T. Alexopoulos et al., Nuclear Instruments and Methods in Physics Research A 640, 110 (2011), 10.1016/j.nima.2011.03.025

[9] T. Behnke, K. Dehmelt et al., JINST 5 (2010) P10011, 10.1088/1748-0221/5/10/P10011

[10] D. Sgalaberna, A. Blondel et al, arXiv:1707.01785

[11] T. Koga et al., JPS Conf. Proc. 8, 023003 (2015), 10.7566/JPSCP.8.023003

[12] C. Betancourt, A. Blondel et al., arXiv:1709.08972

[13] A. Korzenev, Proceeding at EPS-HEP 2017, "Application of SiPM arrays for the readout of a scintillator based time-of-flight detector"

[14] S. Agostinelli, et al., Nuclear Instruments and Methods in Physics Research A506 (2003) 250, 10.1016/S0168-9002(03)01368-8 